Gut, 1979, 20, 467-475

\title{
Morphology of experimental antibiotic-associated enterocolitis in the hamster: a model for human pseudomembranous colitis and antibiotic-associated diarrhoea
}

\author{
A. B. PRICE, H. E. LARSON, AND JULIE CROW \\ From Northwick Park Hospital and Clinical Research Centre, Harrow, Middlesex
}

SUMMARY The morphology of antibiotic-associated enterocolitis in the hamster is described and compared with human antibiotic-associated pseudomembranous colitis. It is shown to be a caecal disease with proliferative mucosal changes and in this respect unlike the human counterpart. The bacteriology and toxicology, however, are identical. In addition, mucosal changes are described in animals on antibiotics but without established enterocolitis. As a result we suggest that there may be a spectrum of human disease ranging from mild antibiotic-associated diarrhoea to established pseudomembranous colitis. Therefore, despite the morphological variation, the hamster remains a good model for investigating the pathogenesis of pseudomembranous colitis and antibiotic-associated enteropathy in general.

Rapid progress in the aetiology of antibioticassociated pseudomembranous colitis (PMC) has followed the intial finding by Larson et al. (1977) of a specific toxin in the stools of affected individuals. The toxin was further identified by showing that its cytopathic effect on cell cultures was neutralised by Clostridium sordellii antitoxin (Larson and Price, 1977; Rifkin et al., 1977) though $C$. sordellii could not te isolated. Next Bartlett et al. (1978) isolated toxigenic $C$. difficile from patients with the disease. Many workers (George et al., 1978b; Larson et al., 1978) since then have confirmed these findings and demonstrated cross-neutralisation between the toxin of $C$. difficile and $C$. sordellii antitoxin (George et al., 1978a). It seems established that proliferation of toxigenic strains of $C$. difficile in the human bowel is one mechanism in the pathogenesis of pseudomembranous colitis.

In parallel with the findings in humans similar results have been produced in Syrian golden hamsters. Small (1968) initially demonstrated that lincomycin produced a fatal enterocolitis when given to hamsters. Because lincomycin and the related clindamycin have been the antibiotics at the centre of a spate of reports on antibiotic-associated pseudomembranous colitis (Cohen et al., 1973; Scott et al.,

Received for publication 25 January 1979
1973), the hamster was the obvious choice as an experimental animal model. $C$. difficile and its toxin have been isolated from hamsters with enterocolitis (Rifkin et al., 1978; Bartlett et al., 1977b) and the organism will reproduce the enterocolitis when reinoculated into susceptible animals (Larson et al., 1978). In this paper we report on the morphology of the hamster disease and compare it with human antibiotic-associated PMC, we also discuss the spectrum of pathology encompassed by the term antibiotic-associated diarrhoea.

\section{Methods}

Syrian hamsters, 1-2 months old, were supplied by the Animal Division, National Institute for Medical Research, Mill Hill, London. Strains of C. difficile isolated from both hamsters and human cases of PMC and identified by standard methods, including gas liquid chromatography of glucose fermentation products(Holdeman and Moore, 1975) were provided by $P$. Honour of the Division of Hospital Infection, C.R.C. All produced toxin in broth culture.

Enterocolitis was produced in the animals by two methods, either by injection of clindamycin, method A, or by oral administration of $C$. difficile, method $B$. The following procedures were used (Table 1). 
Table 1 Summary of treatments

\begin{tabular}{llll}
\hline Method & Group & Antibiotic & $\begin{array}{l}\text { Bacterial } \\
\text { suspension }\end{array}$ \\
\hline A & 1 & Clindamycin i.p. & Nil \\
& 2 & Saline i.p. & Nil \\
B & 1 & Vancomycin p.o. & C. difficile p.o. \\
& 2 & Vancomycin p.o. Sterile broth p.o. & C. difficile p.o. \\
\hline
\end{tabular}

METHOD A

Group A1. Thirty animals were given a single intraperitoneal injection of clindamycin (Upjohns Ltd), $50 \mathrm{mg} / \mathrm{kg}$.

Group A2. Six animals were given $0.5 \mathrm{ml}$ of normal saline intraperitoneally.

\section{METHOD B}

Group B1. Thirty-six animals were given vancomycin (Eli Lilly Co. Ltd), $3 \mathrm{mg} / \mathrm{ml}$, in their drinking water for seven days, then three days of tap water only. They were then force fed $0.5 \mathrm{ml}$ of a toxin-free suspension of $C$. difficile. Twenty animals received a human strain isolated from a case of PMC and 16 a hamster strain isolated from an animal who had succumbed to clindamycin-induced disease.

Group B2. Thirty-six animals were pretreated with vancomycin as in group B1 but then given 0.5 $\mathrm{ml}$ of sterile broth instead of $C$. difficile.

Group B3. Another group of 12 hamsters were given seven days tapwater and then fed $0.5 \mathrm{ml}$ of a toxin-free suspension of $C$. difficile (six a hamster strain, six a human strain).

All animals given vancomycin were kept in exhaustventilated isolators equipped with air filters on both inlets and extracts. Animals were inspected daily for diarrhoea or other evidence of illness. During the experiment animals were killed only if they were severely ill, except that two animals from group B1 and 2 from group B2 were killed daily for four days (16 animals).

Necropsies were carried out on all animals and, as a routine, $5-10 \mathrm{~cm}$ of terminal ileum, the caecum, and $5-10 \mathrm{~cm}$ of colon were pinned out on a cork board and fixed for 24 hours in $10 \%$ formol-saline. Blocks from the ileum, caecum, and colon were taken and standard paraffin embedded, $5 \mu$ haematoxylin and eosin stained sections prepared. A Gram-stain for organisms and a Martius Scarlet Blue stain to demonstrate fibrin were carried out on selected cases. Representative areas of caecum were chosen from both diseased and clinically normal animals for electron microscopic examination. They were fixed in $3 \%$ glutaraldehyde in cacodylate buffer and standard Spurr resin embedded $1 \mu$ and ultra-thin sections cut. The latter were examined on a Phillips EM 300 microscope.
The caecal contents of all animals coming to necropsy were tested for the presence of toxin by methods previously described (Larson and Price, 1977).

\section{Results}

Figure 1 summarises the clinical outcome. Sixty-three per cent of animals given clindamycin intraperitoneally died or were moribund in the first week and $17 \%$ survived for three weeks. No animals receiving vancomycin followed by $C$. difficile survived beyond one week. Sick animals became sluggish and usually remained hunched up in one corner of their cage. Diarrhoea was manifest by wet fur around the anus. However, some animals were found dead without showing prior symptoms or diarrhoea. No deaths occurred in any of the control groups (A2, B2, B3).

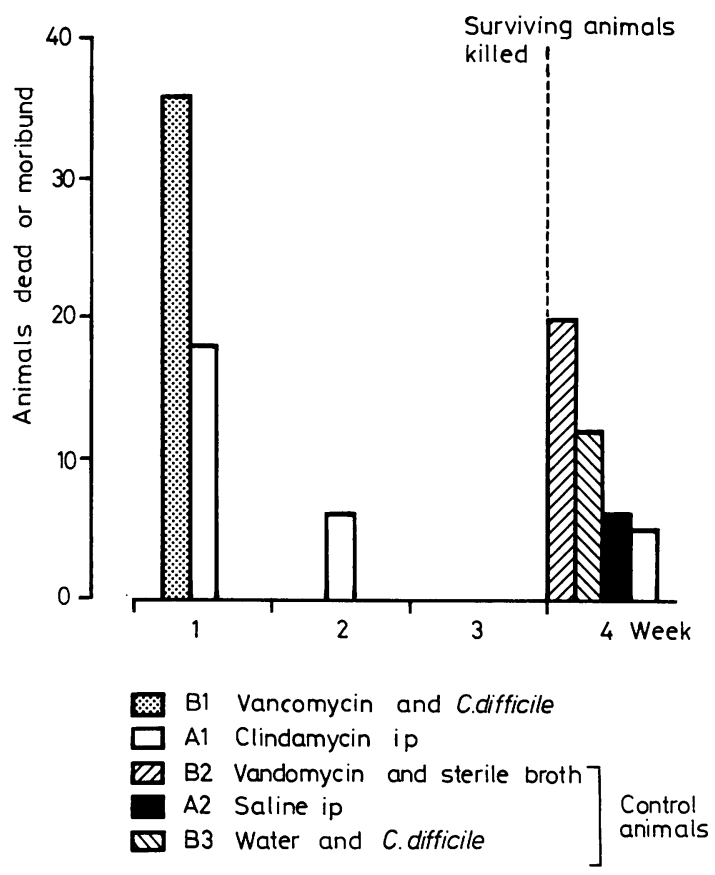

Fig. 1 The outcome of each experimental method.

\section{NECROPSY FINDINGS}

Intestinal disease was the only abnormal finding. Animals with enterocolitis had similar pathology which was independent of the experimental method. Disease invariably involved the caecum, usually the terminal $2-5 \mathrm{~cm}$ of ileum and rarely the proximal colon.

The caecum showed two main patterns of disease that did not comply with any one experimental 
method nor with the time of death. One pattern was of gross distension with widespread haemorrhage (Fig. 2) the caecal contents being fluid and the wall paper-thin. The second pattern showed a caecum with normal or reduced dimensions but having a thickened velvet texture to the mucosa. Haemorrhage, if present, was focal or punctate. Ulceration was noted in isolated cases only, the ulcer floor being covered by a white slough. The gross ileal lesions were those of serosal reddening and mucosal thickening as in the caecum. In the few animals which showed colonic disease serosal congestion was the only macroscopic finding.
The caecums of animals receiving oral vancomycin followed by sterile broth (B2) were also found to be abnormal. They were enlarged and contained semifluid faeces, although there was no gross mural thickening. Animals belonging to groups A2 and B3 were normal.

\section{HISTOPATHOLOGY}

All the animals treated with clindamycin or vancomycin and $C$. difficile who were found dead or moribund invariably had caecal disease. The histological appearances correlated with the two macroscopic patterns noted at necropsy.
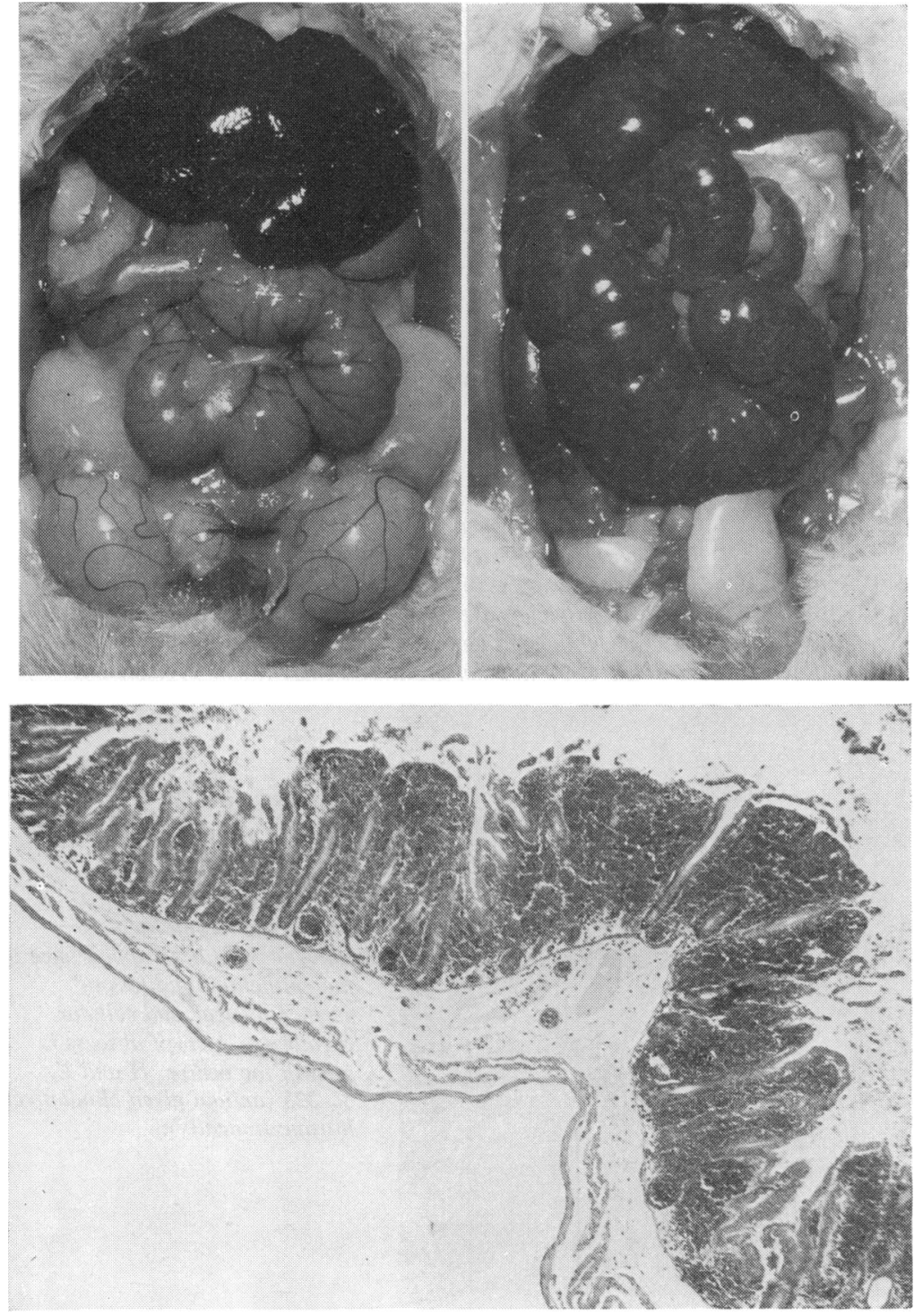

Fig. 2 This compares a normal caecum on the left with, on the right, a dilated haemorrhagic caecum from an animal treated with clindamycin intraperitoneally.

Fig. 3 Caecal mucosa from the diseased animal in Fig. 2 showing diffuse intramucosal haemorrhage. 
The animals with caecal dilatation showed diffuse mucosal haemorrhage. The caecal glands survived in the midst of the haemorrhage (Fig. 3), though in many areas the surface epithelium was lost. Submucosal vascular thrombi were an isolated finding in occasional animals.

The majority of animals showed proliferative changes of the caecal and ileal mucosa with accompanying inflammation (Fig. 4). This was a diffuse change in the caecum and accounted for the velvet texture noted macroscopically. It was accompanied by vascular engorgement and variable amounts of focal haemorrhage. The caecal crypts were elongated and the intercrypt surface epithelium showed florid 'tufting' (Figs 4, 5), the cells having an almost syncytial arrangement. This was in marked contrast to the neat smooth outline of normal caecal mucosa (Fig. 6). At times the proliferation was so florid and villiform it became difficult to distinguish diseased caecum from ileum.

Degenerative changes were also present in the surface cells. The individual enterocytes had lost their brush border, were pale staining, frequently vacuolated, and had enlarged pale nuclei which were no longer situated at the base of the cell.

The mucosal changes were accompanied by a variable mixed inflammatory cell infiltrate in the lamina propria. Polymorphs were seen invading the surface and migrating into glands to form crypt abscesses and a plasma cell infiltrate was a constant feature (Fig. 5). Inflammation was maximal in the mucosa and superficial submucosa but did extend into the muscle coat. Ulceration was noted in occasional cases and when present extended into the submucosa. A surface inflammatory slough covered these ulcers.

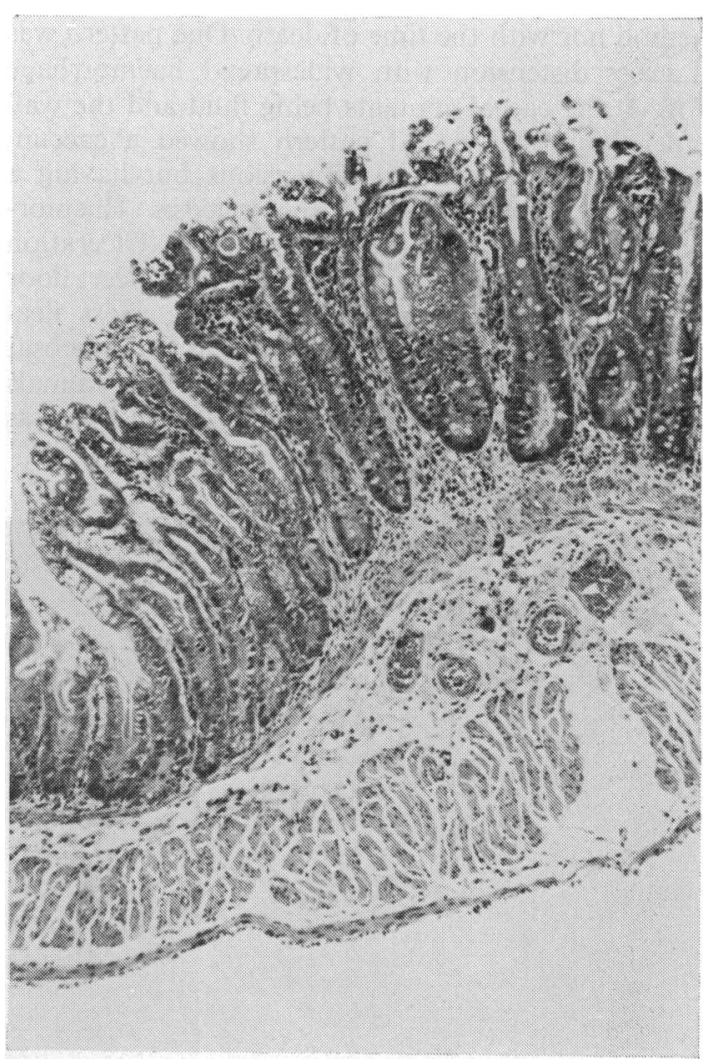

Fig. 4 A caecum showing a greatly thickened mucosa with irregularity of the luminal surface and lengthening of the crypts. Inflammatory cells are present but are not a conspicuous feature. Magnifications quoted in this and the following figures are original magnifications. $H$ and E. $\times 90$ (animal given vancomycin and $\mathrm{C}$. difficile).

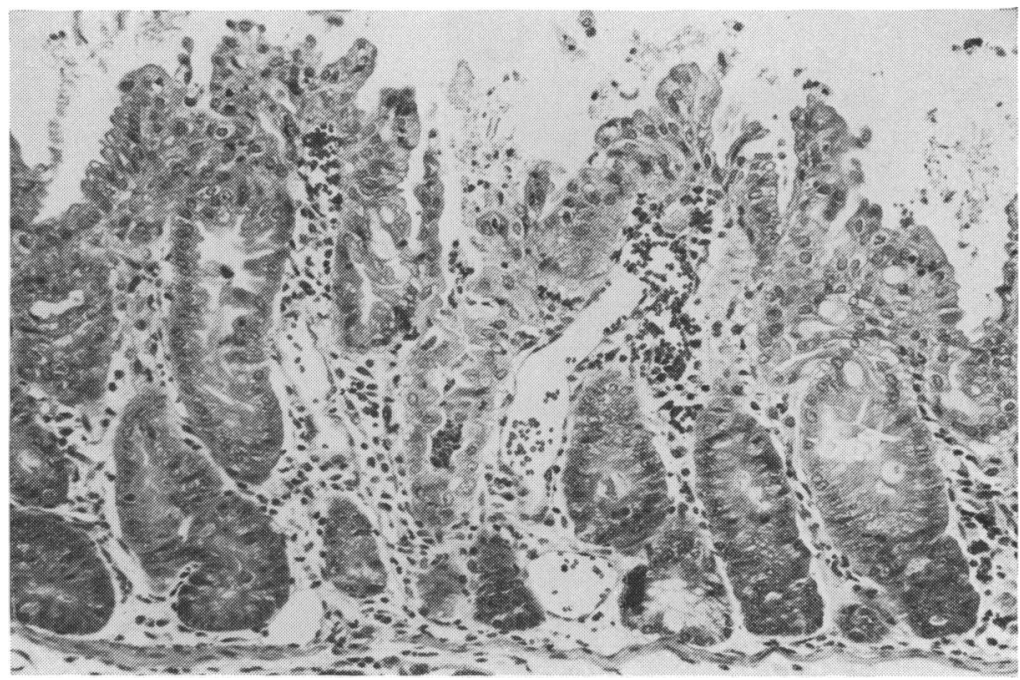

Fig. 5 The caecum of a hamster with enterocolitis showing the tufted appearance of the epithelium and loss of surface nuclear and cellular regularity. A crypt abscess is seen in the centre. $H$ and $E$, $\times 225$ (animal given clindamycin intraperitoneally). 


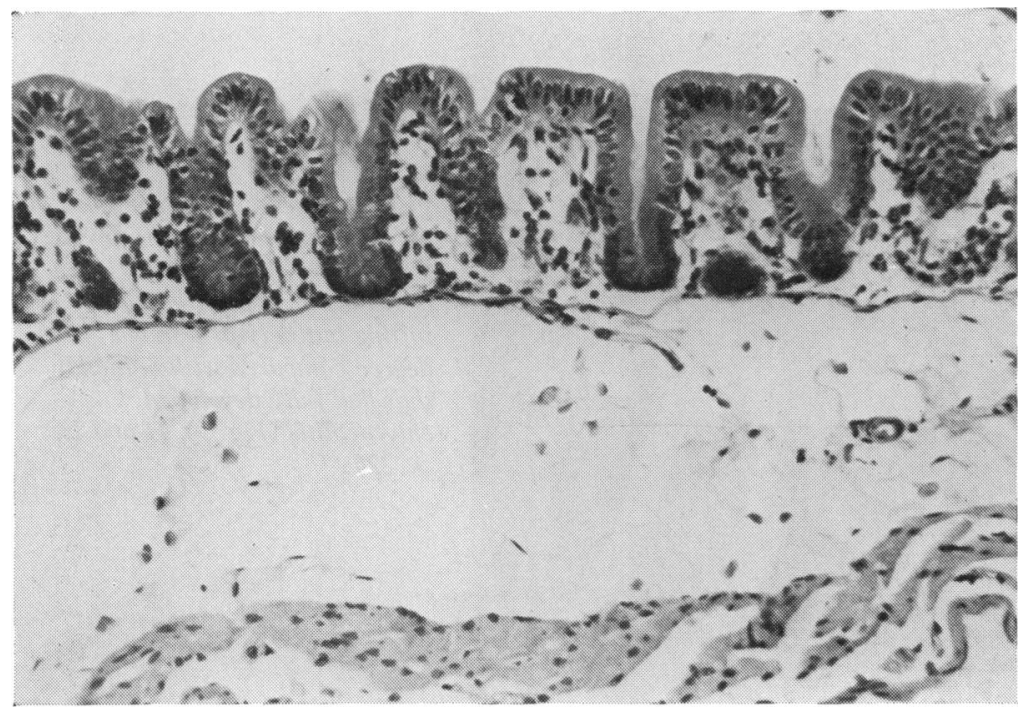

Fig. 6 Normal caecum. The regular surface is a contrast with that seen in animals with toxin positive enterocolitis (Fig. 5) and that in animals treated with vancomycin followed by sterile broth (Fig. 8). $H$ and $E, \times 225$.

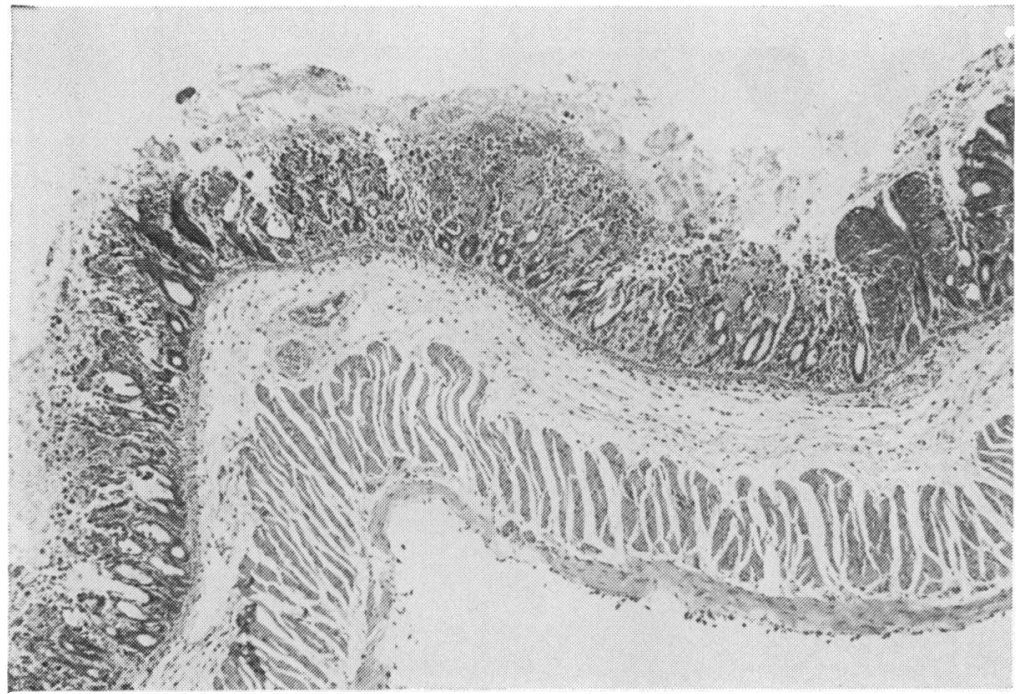

Fig. 7 The caecum from one of only two animals that showed areas of ulceration with 'structured' mucosal necrosis and a fibrinoid-like exudate in the lamina propria. These appearances do resemble those seen in human pseudomembranous colitis but were restricted to the two animals. $H$ and $E, \times 225$ (animal given vancomycin and $\mathrm{C}$. difficile).

In two cases ulceration and 'structured' mucosal necrosis was noted (Fig. 7) with fibrinoid-like material in the lamina propria. The Gram stains showed mixed surface collections of gram positive and negative organisms in all cases. Staining for fibrin showed that only an occasional vessel in isolated cases contained thrombus.

The changes in the ileum were of a similar nature to those in the caecum. With the increase in thickness of the crypts the ratio of villus height to crypt depth decreased and ileum came to resemble caecum in overall architecture. A similar proliferative pattern was noted in the proximal colon in the few cases where it was involved.
Among surviving animals the histopathology was normal except for the animals given vancomycin orally followed by sterile broth. In these the changes were limited to the caecum. The basic normal undulant pattern was preserved but the surface in the intercrypt areas showed heaping up of cells forming bud-like outgrowths and the individual cells had lost their nuclear polarity (Fig. 8). Their staining was variable and cytoplasmic vacuolation was common. The brush border was blurred and the nuclei hyperchromatic and angular. An increase in inflammatory cells in the lamina propria was often present. Having observed this change individual hamsters given oral vancomycin then broth (group 


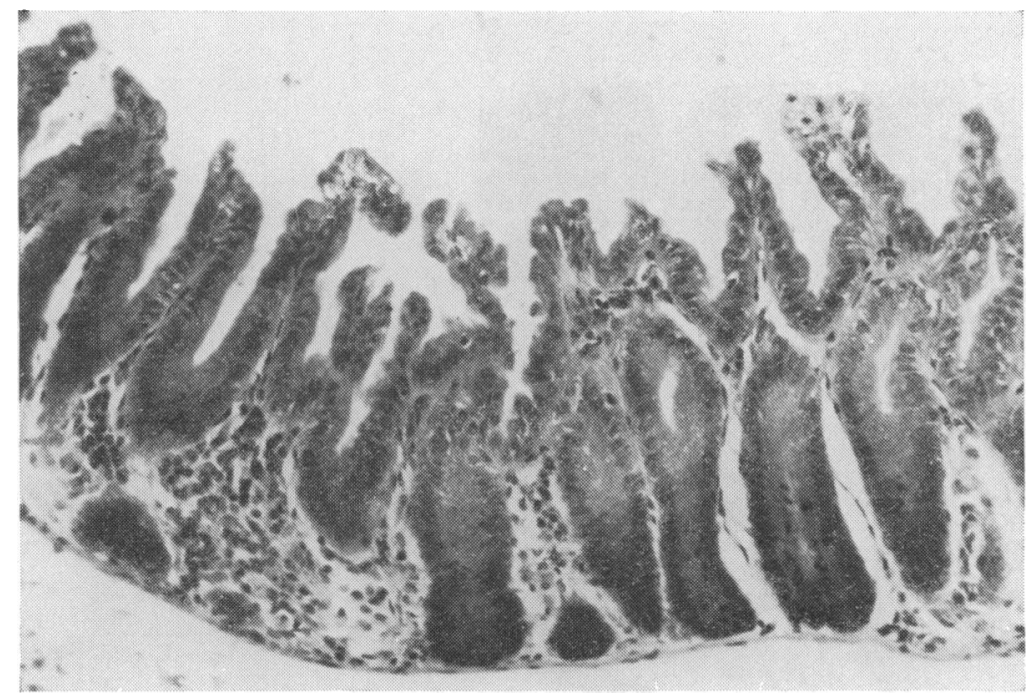

Fig. 8 The caecum from an animal treated with vancomycin followed by sterile broth. There is surface irregularity and tufting but only of a minor degree compared with animals that had fully developed enterocolitis (Fig. 5). $H$ and $E$, $\times 225$.

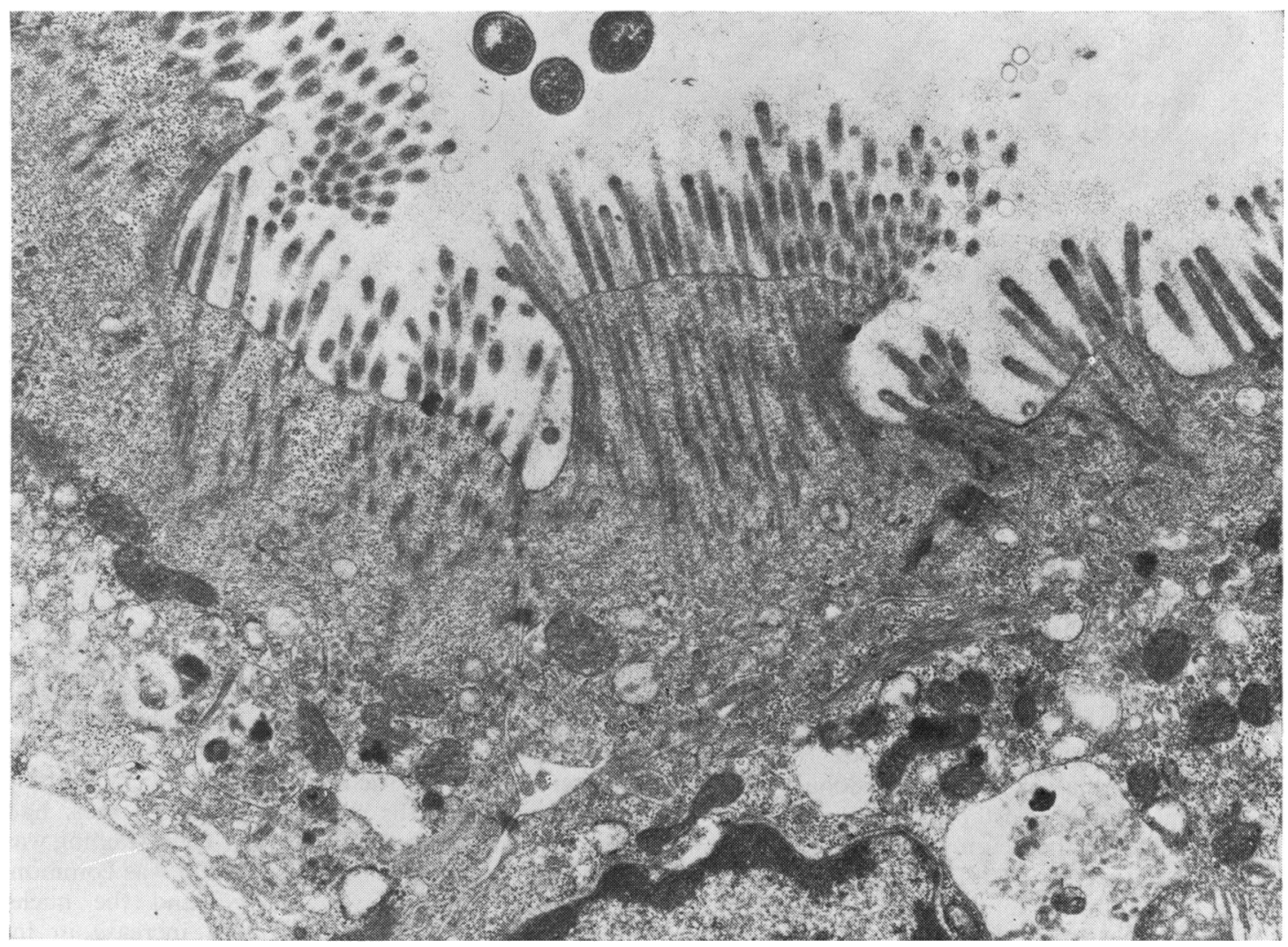

Fig. 9 An electron micrograph of surface caecal mucosa from an animal with enterocolitis treated with vancomycin and $\mathrm{C}$. difficile. Individual cells and their microvilli show irregularity with vacuolar degeneration of the cytoplasm No intracellular organisms can be seen. $\times 16200$. 
B2) were killed on alternate days for 16 days. These changes were maximal immediately after the course of vancomycin and diminished during the next $2 \frac{1}{2}$ weeks.

\section{ELECTRON MICROSCOPY}

Electron microscopic examination of the fully developed proliferative caecal disease showed that the surface epithelial cells were of uneven height and density. The cell surfaces showed marked 'tufting' (Fig. 9) and the micro-villi were irregular and sometimes absent. Degenerative changes were noted in cytoplasmic organelles and large cytoplasmic vacuoles were frequent. Although micro-organisms were seen in the lumen intracellular organisms were not a feature.

In the normal animals occasional vacuolated and degenerating cells were seen but the cell surfaces were smooth and the microvillous border was regular. Sections from an animal in group B2 (oral vancomycin followed by broth), did, however, show some irregularity of the cell surface and microvilli, although the changes were less marked than in those animals with established enterocolitis.

\section{TOXIN ASSAYS}

The caecal contents of all animals inoculated by either method that died or were moribund within the first two weeks contained toxin to a titre of at least $1: 1,000$. Five animals from group A1 survived three weeks (Fig. 1) and when killed no toxin was found. The toxin assays were negative in all the other animals that survived three weeks.

\section{SEQUENTIAL KILLING OF ANIMALS}

INOCULATED BY METHODS B 1 AND B 2

The results are summarised in Table 2 . On day 0 both sets of animals had similar histology but were toxin negative. On day 1 one animal challenged with $C$. difficile had a tiny but definite macroscopic caecal haemorrhage but caecal contents were still toxin negative. Microscopy was similar to the vancomycin-only animals but more widespread and in particular the crypts were beginning to lengthen. By day 2 the animals given $C$. difficile were toxin positive, showed caecal thickening, inflammation and vascular engorgement. By day 3 one animal was dead with florid enterocolitis involving the ileum and caecum.

\section{Discussion}

Unlike other workers (Onderdonk et al., 1977; Lusk et al., 1978), our initial attempts to study clindamycin induced enterocolitis in hamsters were hampered by failure to produce universal
Table 2 Summary of results in experiment involving sequential killing of animals in groups $B 1$ and B2

\begin{tabular}{|c|c|c|c|c|}
\hline \multirow{3}{*}{$\begin{array}{l}\text { Groups } \\
B 1, B 2\end{array}$} & \multicolumn{4}{|c|}{ Vancomycin 7 days then } \\
\hline & \multicolumn{2}{|l|}{ C. difficile } & \multicolumn{2}{|l|}{ Sterile broth } \\
\hline & Morphology & $\begin{array}{l}\text { Toxin } \\
\text { assay }\end{array}$ & Morphology & $\begin{array}{l}\text { Toxin } \\
\text { assay }\end{array}$ \\
\hline $\begin{array}{l}\text { Day } \\
0\end{array}$ & $\begin{array}{l}\text { Intercrypt surface } \\
\text { changes }\end{array}$ & Nega- & & \\
\hline 1 & $\begin{array}{l}\text { Pronounced surface } \\
\text { changes. Crypts } \\
\text { enlarged. Focal } \\
\text { haemorrhage }\end{array}$ & tive & $\begin{array}{l}\text { Intercrypt } \\
\text { surface } \\
\text { changes }\end{array}$ & Negative \\
\hline 2 & $\begin{array}{l}\text { Obvious proliferative } \\
\text { caecitis }\end{array}$ & & & \\
\hline 3 & $\begin{array}{l}\text { Obvious proliferative } \\
\text { caecitis; } 1 \text { animal } \\
\text { dead }\end{array}$ & Positive & & \\
\hline
\end{tabular}

disease (Fig. 1). We thought this happened because some animals were not colonised with $C$. difficile. To produce uniform colonisation we treated animals with vancomycin to eliminate indigenous $C$. difficile and then inoculated this organism by mouth. This technique resulted in a universally fatal outcome (Fig. 1). Pathology and toxin assays show that both methods, clindamycin intraperitoneally and vancomycin orally followed by $C$. difficile, produce an identical enterocolitis. $C$. difficile inoculation without prior antibiotic treatment had no effect and animals given vancomycin alone then kept free from any clostridial exposure also survived and remained toxin negative (Fig. 1). However, to ensure these latter conditions the animals had to be housed in isolators.

Several comparisons with human pseudomembranous colitis are suggested. First, human derived strains of $C$. difficile induce the same disease in hamsters as the hamster derived strains. Second, the necessity for antibiotic pretreatment mimics most human situations. Thirdly, the association and properties of the toxin with established enterocolitis is similar in both (Bartlett et al., 1977b; Rifkin et al., 1978). However, unlike human antibiotic-associated pseudomembranous colitis, enterocolitis in the hamster invariably occurred in the caecum, often in the terminal ileum, but rarely in the colon. So different is the physiological role of the caecum in the hamster from that in the human that an anatomical variation in the pathology is not too surprising.

But what is difficult to explain are the markedly different histological changes. We stress that a membrane or plaque is rarely found in hamster disease (Fig. 7) (Bartlett et al., 1977a; Lusk et al., 1978). The characteristic histology was either 
mucosal epithelial proliferation accompanied by surface degenerative changes (Figs 4,5 ) or mucosal haemorrhage (Fig. 3), and not the focal superficial crypt necrosis with exudation, typical of human pseudomembranous colitis (Price and Davies, 1977). When ulceration was present in the hamster caecum the covering inflammatory debris was considered to be non-specific.

It is not clear how $C$. difficile infection produces these changes. Gram stains and electron microscopy failed to show organisms invading the mucosa and this is also true for human pseudomembranous colitis (Steer, 1975). Oral administration of the toxin does not produce lesions (unpublished data), while intraperitoneal injection produces fatal systemic toxicity with widespread haemorrhagic lesions (Rifkin et al., 1978). Intracaecal injections of toxin have been reported to give caecal lesions (Bartlett et al., 1977b) but in our experience with this method the animals die within 24 hours from the systemic effects. Administration of large amounts of $C$. sordellii antitoxin protects hamsters from death after clindamycin challenge but the histological findings have not been reported (Rifkin et al., 1978). Certain clostridial toxins are known to be vasoconstrictors and might induce ischaemic damage (Marston, 1977). This would account for the haemorrhagic element seen in the enterocolitis of the hamster and for the similarity of human pseudomembranous to ischaemic colitis. Alternatively, the toxin may have a direct mucosal effect (McDonel and Duncan, 1975) that would explain the initial small necrotic foci seen in the human disease (Price and Davies, 1977). However, neither mechanism satisfactorily explains the proliferative lesions described in the hamster model. Mucosal proliferation as a response to bacterial infection is unusual in humans but recognised in certain animal species. It is seen in wet-tail (proliferative ileitis) a common hamster intestinal infection with many similarities to the enteritis seen here (Jacoby, 1978), and it also occurs in forms of swine dysentery (Glock et al., 1974). Surprisingly the administration of vancomycin alone caused mild caecal changes (Fig. 8). In the most florid examples the pattern was difficult to distinguish from that of mild toxin positive enteritis, but the caecal contents remained toxin negative. The histological patterns diverged in parallel with the appearances of toxin as seen in the experiment where the animals were killed at daily intervals (Table 2). It is tempting to postulate that the morphological changes produced by clindamycin, vancomycin followed by $C$. difficile, and vancomycin alone (Figs 5, 8) are part of a single spectrum. This graded response to antibiotics might have a close human parallel. It is well established that certain patients who receive antibiotics develop diarrhoea (Tedesco, 1975); it is not known if there are any mucosal abnormalities at this stage. A minority of patients in this 'susceptible' state go on to develop a definite colitis which is either nonspecific or pseudomembranous in type (Gibson et al., 1975). It is in the latter group that $C$. difficile is found in the gut.

It is also important to know whether both the human and animal disease are endogenous or exogenous infections. The spectrum of proliferative histological changes suggest a gradual development perhaps associated with an endogenous infection. However, the animals given vancomycin alone remained well if not exposed to the organism (Fig. 1), a feature of particular importance to clinical management since vancomycin is used to treat human pseudomembranous colitis(Tedesco et al., 1978). So far, work in humans suggests that $C$.difficile is not an endogenous member of the normal adult flora (Larson et al., 1978) but more extensive epidemiological studies are needed. Exogenous infection after clindamycin would also best explain the results shown in Fig. 1 .

Our findings suggest that the hamster, despite certain morphological differences, is still a good model in which to study the wider spectrum of antibiotic-associated intestinal enteropathy, in particular the role of various antibiotics in the spectrum of clinical disease encompassing diarrhoea, nonspecific colitis, and pseudomembranous colitis. It is also a model for the conditions that allow $C$. difficile to become established as an intestinal pathogen.

We would like to thank the following for technical help: Chris Sowter, David Gunner, Sheila Holt, the Animal Histopathology Department, and the Division of Comparative Medicine. We would also like to thank John Clark for the photography and Jillian Jones for the preparation of the manuscript.

\section{References}

Bartlett, J. G., Chang, T. W., Gurwith, M., Gorbach, S. L., and Onderdonk, A. B. (1978). Antibiotic-associated pseudomembranous colitis due to toxin-producing Clostridia. New England Journal of Medicine, 298, 531-534.

Bartlett, J. G., Onderdonk, A. B., and Cisneros, R. L. (1977a). Clindamycin-associated colitis in hamsters: protection with vancomycin. Gastroenterology, 73, 772-776.

Bartlett, J. G., Onderdonk, A. B., Cisneros, R. L., and Kasper, D. L. (1977b). Clindamycin-associated colitis due to a toxin-producing species of clostridium in hamsters. Journal of Infectious Diseases, 136, 701-705.

Cohen, L. E., McNeill, C. J., and Wells, R. F. (1973) Clindamycin-associated colitis. Journal of the American Medical Association, 223, 1379-1380.

George, W. L., Sutter, V. L., Goldstein, E. J. C., Ludwig, S. L., and Finegold, S. M. (1978a). Aetiology of anti- 
microbial-agent-associated colitis. Lancet, 1, 802-803.

George, R. M., Symonds, J. M., Dimock, F., Brown, J. D., Arabi, Y., Shinagawa, N., Keighley, M. R. B., AlexanderWilliams, J., and Burdon, D. W. (1978b). Identification of Clostridium difficile as a cause of pseudomembranous colitis. British Medical Journal, 1, 695.

Gibson, G. E., Rowland, R., and Hecker, R. (1975). Diarrhoea and colitis associated with antibiotic treatment. Australian and New Zealand Journal of Medicine, 5, 340-347.

Glock, R. D., Harris, D L., and Kluge, J. P. (1974). Localization of spirochetes with the structural characteristics of Treponema hyodysenteriae in the lesions of swine dysentery. Infection and Immunity, 9, 167-178.

Holdeman, L. V., and Moore, W. E. C. (1975). Anaerobe Laboratory Manual. Virginia Polytechnic Institute; Blacksburg, Virginia.

Jacoby, R. O. (1978). Transmissible ileal hyperplasia of hamsters. American Journal of Pathology, 91, 433-450.

Larson, H. E., Parry, J. V., Price, A. B., Davies, D. R., Dolby, J., and Tyrell, D. A. J. (1977). Undescribed toxin in pseudomembranous colitis. British Medical Journal, 1, 1246-1248.

Larson, H. E., and Price, A. B. (1977). Pseudomembranous colitis: Presence of Clostridial toxin. Lancet, 2, 13121314.

Larson, H. E., Price, A. B., Honour, P., and Borriello, S. P. (1978). Clostridium difflcile and the aetiology of pseudomembranous colitis. Lancet, 1, 1063-1066.

Lusk, R. H., Fekety, R., Silva, J., Browne, R. A., Ringler, D. H., and Abrams, G. D. (1978). Clindamycin-induced enterocolitis in hamsters. Journal of Infectious Diseases,
137, 464-475.

McDonel, J. L., and Duncan, C. L. (1975). Histopathological effect of Clostridium perfringens enterotoxin in the rabbit ileum. Infection and Immunity, 12, 1214-1218.

Marston, A. (1977). Intestinal Ischaemia. Edward Arnold: London.

Onderdonk, A. B., Hermos, J. A., and Bartlett, J. (1977). The role of intestinal microflora in experimental colitis. American Journal of Clinical Nutrition, 30, 1819-1825.

Price, A. B., and Davies, D. R. (1977). Pseudomembranous colitis. Journal of Clinical Pathology, 30, 1-12.

Rifkin, G. D., Fekety, F. R., and Silva, J., Jr. (1977). Antibiotic-induced colitis implication of a toxin neutralised by Clostridium sordellii antitoxin. Lancet, 2, 1103-1106.

Rifkin, G. D., Fekety, F. R., and Silva, J. (1978). Neutralization by Clostridium sordellii antitoxin of toxins implicated in clindamycin-induced cecitis in the hamster. Gastroenterology, 75, 422-424.

Scott, A. J., Nicholson, G. I., and Kerr, A. R. (1973). Lincomycin as a cause of pseudomembranous colitis. Lancet, 2, 1232-1234.

Small, J. D. (1968). Fatal enterocolitis in hamsters given lincomycin hydrochloride. Laboratory Animal Care, 18, 411-420.

Steer, H. W. (1975). The pseudomembranous colitis associated with clindamycin therapy-a viral colitis. Gut, 16, 695-706.

Tedesco, F. J. (1975). Ampicillin-associated diarrhoea-A prospective study. American Journal of Digestive Diseases, 20, 295-297.

Tedesco, F., Markham, R., Gurwith, M., Christie, D., and Bartlett, J. G. (1978). Oral vancomycin for antibioticassociated pseudomembranous colitis. Lancet, 2, 226-228. 